\title{
JETDE 2011 Preface
}

Barbara B. Lockee

It is with great honor that I introduce this annual issue of the Journal of Educational Technology and Development Exchange. This collection of papers reflects an excellent compendium of research across a variety of topics, though all are bound by a single factor - the authors' collective interest in contributing information in support of the improvement of teaching and learning. Some papers feature the development of new techniques for application within existing technological environments, while some papers detail the creation of new tools and unique contexts for learning. Hopefully, the following overview demonstrates the outstanding scholarly work included in this issue of JETDE.

Innovations in learning environments can support new ways of teaching and learning. As authors Jeff J. S. Huang, Stephen J. H. Yang, and Matt C. W. Chang indicate in their paper, "The Effect of ePortfolio Satisfaction on Students' Learning Motivation and Internet Self-Efficacy", the development of such environments can be a very costly and time-intensive endeavor. Their examination of the influences of learner motivation, Internet self-efficacy, and ePortfolio satisfaction on the use of ePortfolios provides an insightful look at the interactions among these variables related to learner reactions to this alternative assessment approach.

In their inquiry focused on English as a Second Language (ESL) learning, Hong-Fa Ho and Chen Huong, emphasize the importance of determining the extent of the learner's ESL vocabulary. Their article, "A Multiple Aspects Quantitative Indicator for Ability of English Vocabulary: Vocabulary Quotient", describes their creative development of the measure of a learner's vocabulary quotient (VQ), comprised of spelling, reading comprehension, listening comprehension, and spelling selection. This measurement tool shows positive steps toward correlating facets of language learning, providing important implications for instructional design for ESL experiences.

Author Ai Chun Yen details the use of an e-story mapping system within an LMS in the paper entitled, "Academic Reading to Academic Writing Via Learning Management System". The study also introduces the "4P model" - plan-produce-publish-present, as a means to provide context to the reading content, followed with the development of academic writing skills. This strategy based on constructivist theory helps enhance English language learning, as well as classic literature awareness, through a collaborative and empirically-based instructional strategy.

Regarding collaborative learning strategies, authors Cheng-Ting Chen and Kuo-Chen Li detail the process and positive outcomes of using student-produced video as an effective instructional method to teach English to non-English speaking students in Taiwan. The paper "Action to Acquisition-Boost Students' English Learning Motivation with Filmmaking Project" exemplifies the benefits of project-based learning, as students reacted to this approach with great enthusiasm, indicating an increase in motivation and an appreciation for a meaningful learning experience that could be translated to real-world application.

The integration of technology for learning relies on a variety of factors, one of which is a positive teacher disposition toward the use of technological innovations in the classroom. In 
their paper, "Helping Teachers Develop Positive Dispositions about Technology-based Learning: What A Brief Global Learning Project Revealed", Carol A. Smith, Heather Schugar, and Cynthia A. Moyer demonstrate the effectiveness of teaching through technology instead of about technology, and how such a focus can both increase teacher awareness of new strategies for mediated learning and contribute to a positive shift in disposition about technology integration.

Second language learning research has proven the importance of providing authentic contexts for the application of the target language. Authors Charles Xiaoxue Wang, Mary L. Lefaiver, Christy Hunt, and Qi Wang validate this principle through a creative and unique virtual world learning environment in their paper, "Teaching in an EFL Program in Second Life: Student Teachers' Perspectives and Implications". The results of this study indicate that student teachers, the learner group in this case, particularly value of the ability to interact with multiple learners in meaningful situations, therefore increasing the potential for the acquisition and transfer of the new language.

While the use of games for learning has been greatly advocated in recent literature, Timothy Dedeaux and Taralynn Hartsell discuss how such games may vary in terms of their educational impact. In their paper, "Educational Computer Games and Spanish Content Learning”, these authors describe a preliminary investigation in the use of games for second language instruction, the early results of which set the stage for further exploration of how differing pedagogies incorporated into educational games may have differing influences on student motivation and learning.

While much recent research related to the use of technology for instruction tends to feature the most current innovations, there are advantages to the examination of new approaches to the application of well-adopted and readily available technologies. Feihong Wang, Xin Chen, and Weini Fang, in their paper "Integrating Cell Phones into a Chinese High School EFL Classroom: Students' Attitudes, Technological Readiness, and Perceived Learning", show how a popular communication device such as a cell phone can be implemented into the ESL classroom with positive results.

Meanwhile, some technological advancements are beginning to see potential in terms of supporting a broad array of instructional needs. The paper, "Augmented Reality: An Overview and Five Directions for AR in Education", written by Steve Chi-Yin Yuen, Gallayanee Yaoyuneyong, and Erik Johnson, provides an excellent overview of how the use of augmented reality can address a wide variety of learning and performance improvement outcomes.

The proliferation of online learning is readily supported by the ever-increasing availability of a wide variety of communication tools for use in distance-delivered educational programs. Author Shuyan Wang explores student and instructor perceptions of such tools in the paper, "Promoting Students' Online Engagement with Communication Tools". Findings related to student use of these tools, as well as their reflections on the benefits and challenges related to each type, offer informative guidance for those instructors considering the design of communication strategies for online learning experiences.

One of the advantages I have in the reviewing these manuscripts is the ability to convey to others how the work of SICET scholars supports the application of research outcomes to practice. It is clear that the investigations and developmental analyses included in this issue have pragmatic outcomes that can guide practitioners toward the development of effective instruction and positive learning experiences. I very much appreciate this learning opportunity! 